\title{
INDEX OF FAITHFUL NORMAL CONDITIONAL EXPECTATIONS
}

\author{
SZE-KAI TSUI
}

(Communicated by Paul S. Muhly)

\begin{abstract}
Let $E$ be a faithful normal conditional expectation of a factor $M$ onto its subfactor $N$, and the index of $E$ be denoted by $\mathrm{IND}_{E}$. We investigate the question: For two such faithful normal conditional expectations $E_{1}, E_{2}$ of $M$ onto $N$, when does $\mathrm{IND}_{E_{1}}=\mathrm{IND}_{E_{2}}$ hold? In this paper we answer this question completely for type $I$ factor $M$. We also derive a tensor product formula for index, i.e., $\mathrm{IND}_{E_{1} \otimes E_{2}}=\left(\mathrm{IND}_{E_{1}}\right)\left(\mathrm{IND}_{E_{2}}\right)$. For any $\alpha>9$ we construct uncountable nonisomorphic faithful normal conditional expectations $E$ of a factor $M$ onto its subfactor $N$ with $\mathrm{IND}_{E}=\alpha$ such that both of $M$ and $N$, are of type $I$ or $I I$ or $I I I_{\lambda}, 0 \leq \lambda \leq 1$. For each $\beta \in\left\{4 \cos ^{2} \pi / n, \mid n \geq 3\right\} \cup[4, \infty)$ we exhibit a type $I I I_{\lambda}$ factor $M$ and its subfactor $N$ and a faithful normal conditional expectation $E$ such that $\mathrm{IND}_{E}=\beta$.
\end{abstract}

\section{INTRODUCTION}

In 1984 Kosaki generalized the definition of index to arbitrary factors $M$ and their subfactors $N$ in case of the existence of a faithful normal conditional expectation $E$ of $M$ onto $N$ [6]. The index of $E$, denoted by $\mathrm{IND}_{E}$, is defined as $E^{-1}(I)$. Then the natural question to ask is "for what pairs of $E_{1}, E_{2}$ do we have $\mathrm{IND}_{E_{1}}=\mathrm{IND}_{E_{2}}$ ?" In $\S 2$ of this article we answer this question completely for type $I$ factors $M$ (see 2.8). In general, we are able to exhibit uncountable nonouterconjugate faithful normal conditional expectations of a factor onto its subfactor, (both of which can be of type I, II, or III) with the same index greater than or equal to 9 (see 3.12). The values of indices in our example can not be less than 4 , for, if $\mathrm{IND}_{E}=4 \cos ^{2} \pi / n$ for $n \geq 3$, by a theorem due to Haagerup [4], there is only one faithful normal conditional expectation of $M$ onto its subfactor $N$. Recently, similar works on uncountable conjugacy classes of *-endomorphisms with the same index were done by Powers [8], Choda [2], and Bures-Yin [1]. One major tool used in deriving the above result is the formula for the index of the tensor product of two faithful normal conditional expectations. It states that for any $E_{i}$, faithful normal conditional expectations

Received by the editors August 7, 1989.

1980 Mathematics Subject Classification (1985 Revision). Primary 46L10, 46L35.

Key words and phrases. Index of faithful normal conditional expectations, index of tensor products, spatial derivatives, relative commutants. 
of a factor $M_{i}$ onto its subfactor $N_{i}, i=1,2, \mathrm{IND}_{E_{1} \otimes E_{2}}=\left(\mathrm{IND}_{E_{1}}\right)\left(\mathrm{IND}_{E_{2}}\right)$, where $E_{1} \otimes E_{2}$ is a faithful normal conditional expectation of $M_{1} \otimes M_{2}$ onto $N_{1} \otimes N_{2}$ (see Theorem 3.11). In the case when $M_{i}, N_{i}$ are factors of type II $_{1}$ and $E_{i}$ are trace preserving, the result can be easily verified as in [5]. Using this theorem we can easily construct type III factors $M, N$ and a faithful normal conditional expectation $E$ of $M$ onto $N$ such that $\operatorname{IND}_{E}$ can be any number in $\left\{4 \cos ^{2} \pi / n \mid n \geq 3\right\} \cup[4, \infty)$ (see 3.10).

\section{INDEX OF TYPE I FACTORS}

Let $M, N$ be type $I$ factors and $N \subseteq M$. In this section we completely describe all faithful normal conditional expectations $E$ from $M$ onto $N$ and their indices. Hence we may answer the following question completely. For $E_{1}, E_{2}$, normal faithful conditional expectations of $M$ onto $N$, when does $\mathrm{IND}_{E_{1}}=\mathrm{IND}_{E_{2}}$ hold?

2.1. Let $M=B(\mathscr{H})$, the von Neumann algebra of all bounded linear operators on a Hilbert space $\mathscr{H}$, and $N=\mathscr{C}$, where $\mathscr{H}$ is separable (may be of finite dimension) and $\mathscr{C}$ is the complex field. Any normal state $\varphi$ on $B(\mathscr{H})$ is of the form $\varphi(x)=\operatorname{tr}(A x)$ for some positive trace class operator $A$ with $\operatorname{tr}(A)=1$, where $\operatorname{tr}$ is the trace on $B(\mathscr{H})$. Let $\left\{\lambda_{i}\right\}$ be the set of all eigenvalues of $A$. If $\varphi$ is faithful, then $\lambda_{i}>0$ for all $i$. In this notation we have the following.

2.2. Proposition. $\mathrm{IND}_{\varphi}=\sum_{i=1}^{\infty} 1 / \lambda_{i}$ for any faithful normal state $\varphi$ on $B(\mathscr{H})$. In particular $\mathrm{IND}_{\varphi}$ is finite if and only if $\mathscr{H}$ is of finite dimension.

Proof. Let $N_{\varphi}=\left\{x \in M \mid \varphi\left(x^{*} x\right)<\infty\right\}, \mathscr{H}_{\varphi}$ be the Hilbert space completion of $\widehat{N}_{\varphi}$ with denoting the embedding of $N_{\varphi}$ into $\mathscr{H}_{\varphi}$ and $\pi_{\varphi}$ the GNS representation induced by $\varphi$. As in [3], for each vector $\xi \in \mathscr{H}^{\varphi}$, we define densely defined operator $R^{\varphi}(\xi)$ from $\mathscr{H}_{\varphi}$ to $\mathscr{H}$ by $R^{\varphi}(\xi)\left({ }^{\wedge} x\right)=x \xi, \forall x \in N_{\varphi}$. If $\xi=A^{1 / 2} \zeta$ for some $\zeta \in \mathscr{H}$, with $\|\zeta\|=1$, then $\left\|^{\wedge}(x)\right\|^{2}=\varphi\left(x^{*} x\right)=$ $\operatorname{tr}\left(A^{1 / 2} x^{x} x A^{1 / 2}\right) \geq\left\|\left(x A^{1 / 2}\right)^{*}\left(x A^{1 / 2}\right)\right\|=\left\|x A^{1 / 2}\right\|^{2} \geq\left\|x A^{1 / 2} \zeta\right\|^{2}=\|x \xi\|^{2}$ and $R^{\varphi}(\xi)$ is bounded. For those $\xi$ 's we denote $R^{\varphi}(\xi) R^{\varphi}(\xi)^{*}$ by $\theta^{\varphi}(\xi, \xi)$. It is easily checked that $\theta^{\varphi}(\xi, \xi) \in M_{+}$. Let $\left\{\xi_{i}\right\}$ be the complete orthonormal set of eigenvectors of $A$ corresponding to $\left\{\lambda_{i}\right\}$, and $\xi_{i} \odot \xi_{j}$ be the rank-one operation in $B(\mathscr{H})$ such that $\left(\xi_{i} \odot \xi_{j}\right)(\xi)=\left\langle\xi, \xi_{i}\right\rangle \xi_{j}$ for $\xi \in \mathscr{H}$. We claim that $\theta^{\varphi}\left(\xi_{i}, \xi_{i}\right)=I_{\mathscr{H}} / \lambda_{i}$. For, we have

$$
R^{\varphi}\left(\xi_{i}\right)\left({ }^{\wedge}\left(\xi_{k} \odot \xi_{l}\right)\right)=\left(\xi_{k} \odot \xi_{l}\right)\left(\xi_{i}\right)=\left\langle\xi_{i}, \xi_{k}\right) \xi_{l}=\delta_{i, k} \xi_{l},
$$

and

$$
\begin{aligned}
\left\langle R^{\varphi}\left(\xi_{i}\right)^{*}\left(\xi_{j}\right),{ }^{\wedge}\left(\xi_{k} \odot \xi_{l}\right)\right\rangle & =\left\langle\xi_{j}, R^{\varphi}\left(\xi_{i}\right)\left({ }^{\wedge}\left(\xi_{k} \odot \xi_{l}\right)\right)\right\rangle \\
& =\left\langle\xi_{j}, \delta_{i, k} \xi_{l}\right\rangle=\delta_{i, k} \delta_{j, l} .
\end{aligned}
$$

Since ${ }^{-}\left(\xi_{k} \odot \xi_{l}\right)$ form an orthogonal basis for $\mathscr{H}_{\varphi}$, and

$$
\begin{aligned}
\left\|^{\wedge}\left(\xi_{i} \odot \xi_{j}\right)\right\|^{2} & =\varphi\left(\left(\xi_{i} \odot \xi_{j}\right)^{*}\left(\xi_{i} \odot \xi_{j}\right)\right) \\
& =\operatorname{tr}\left(A\left(\xi_{i} \odot \xi_{i}\right)\right)=\left\langle A \xi_{i}, \xi_{i}\right\rangle=\lambda_{i},
\end{aligned}
$$


we get $R^{\varphi}\left(\xi_{i}\right)^{*}\left(\xi_{j}\right)={ }^{\wedge}\left(\xi_{i} \odot \xi_{j}\right) / \lambda_{i}$ and hence $R^{\varphi}\left(\xi_{i}\right) R^{\varphi}\left(\xi_{i}\right)^{*}\left(\xi_{j}\right)=\left(1 / \lambda_{i}\right) \xi_{j}$ $\forall i, j=1,2, \ldots$. Thus $\theta^{\varphi}\left(\xi_{i}, \xi_{i}\right)=I_{\mathscr{P}} / \lambda_{i}$, and

$$
\begin{aligned}
\operatorname{Ind}_{\varphi}=\varphi^{-1}(I) & =\varphi^{-1}\left(\sum_{i=1}^{\infty}\left(\xi_{i} \odot \xi_{i}\right)\right) \\
& =\sum_{i=1}^{\infty} \varphi^{-1}\left(\xi_{i} \odot \xi_{i}\right) \\
& =\sum_{i=1}^{\infty} \theta^{\varphi}\left(\xi_{i}, \xi_{i}\right) \\
& =\left(\sum_{i=1}^{\infty} 1 / \lambda_{i}\right) I_{\mathscr{H}} .
\end{aligned}
$$

The second to the last equality, which was first used in [6], can be easily verified. Furthermore since $\sum \lambda_{i}=1, \lambda_{i}>0$, we have $\operatorname{IND}_{\varphi}=\infty$ unless $\left\{\lambda_{i}\right\}$ is a finite set, or, equivalently $\mathscr{H}$ is of finite dimension $n$. In that case the minimum $\left\{\operatorname{IND}_{\varphi} \mid \varphi\right\}=n^{2}$. Q.E.D.

The next theorem is due to Murray and von Neumann as Theorem 8.6.1 in [7].

2.3. Theorem. Let $N$ be a factor of type $I$ in $B(\mathscr{H})$ for a separable Hilbert space $\mathscr{H}$. Then $N \simeq B\left(\mathscr{H}_{1}\right)$ for a subspace $\mathscr{H}_{1}$ of $\mathscr{H}$, and $\mathscr{H} \cong \mathscr{H}_{1} \otimes \mathscr{H}_{2}$ with $B(\mathscr{H}) \cong B\left(\mathscr{H}_{1}\right) \otimes B\left(\mathscr{H}_{2}\right), N^{\prime} \simeq B\left(\mathscr{H}_{2}\right)$.

In the notation as in Theorem 2.3, we have:

2.4. Proposition. The only normal conditional expectation $E$ of $B(\mathscr{H})$ onto a subfactor $N$ of type $I$ is id $\otimes \varphi$, where $\varphi$ is a normal state on $B\left(\mathscr{H}_{2}\right)$, and id is the identity map on $B\left(\mathscr{H}_{1}\right)$.

Proof. Denote a linear functional $\varphi$ on $B\left(\mathscr{H}_{2}\right)$ by $\varphi(y)=E(I \otimes y)$ which is well defined. In fact, because $E(I \otimes y) \in B\left(\mathscr{H}_{1}\right) \otimes I$ and $E((x \otimes I)(I \otimes y))=$ $(x \otimes I) E(I \otimes y)=E((I \otimes y)(x \otimes I))=E(I \otimes y)(x \otimes I)$, we have $E(I \otimes y) \in$ $Z\left(B\left(\mathscr{H}_{1}\right) \otimes I\right)$, the center of $B\left(\mathscr{H}_{1}\right) \otimes I$, and hence $E(I \otimes y)=$ scalar $\cdot(I \otimes I)$. Then $\varphi$ is a state, for $\varphi(I \otimes I)=(I \otimes I)$ and $\varphi$ is positive. Then $\varphi$ is also normal, for $E$ is. Finally

$$
\begin{aligned}
E\left(\sum x_{i} \otimes y_{i}\right) & =\sum E\left(\left(x_{i} \otimes I\right)\left(I \otimes y_{i}\right)\right) \\
& =\sum\left(x_{i} \otimes I\right) \varphi\left(y_{i}\right)=\sum x_{i} \otimes \varphi\left(y_{i}\right) I \\
& =\sum(\operatorname{id} \otimes \varphi)\left(x_{i} \otimes y_{i}\right)=(\operatorname{id} \otimes \varphi) \sum x_{i} \otimes y_{i}, \\
& \forall x_{i} \in B\left(\mathscr{H}_{1}\right), y_{i} \in B\left(\mathscr{H}_{2}\right) . \quad \text { Q.E.D. }
\end{aligned}
$$

In the same notation as in Theorem 2.3, let $E$ be a faithful normal conditional expectation of $B(\mathscr{H})$ onto $B\left(\mathscr{H}_{1}\right)$ and $E=\mathrm{id} \otimes \varphi$. Identifying $B\left(\mathscr{H}_{1}\right)^{\prime}$ 
with $B\left(\mathscr{H}_{2}\right)$, we show

2.5. Theorem. $E^{-1}=\varphi^{-1}$.

Proof. Let $\psi$ be an arbitrary faithful normal state on $N$ and $l$ be the identity state on $\mathscr{C}$. As in [6], $E^{-1}$, an operator valued weight from $N^{\prime}$ to $M^{\prime}$, is characterized by $\Delta(\psi \circ E \mid l)=\Delta\left(\psi \mid \imath \circ E^{-1}\right)$, where $\Delta$ denotes the spatial derivative defined by Connes in [3]. Since the above equation completely determines $E^{-1}$ for given $E$, we need only to show the following $\Delta(\psi \circ E \mid l)=\Delta\left(\psi \mid \imath \circ \varphi^{-1}\right)$. In fact, for $\eta \in \mathscr{H}$,

$$
\begin{aligned}
\left\|\left[\Delta\left(\psi \mid l \circ \varphi^{-1}\right)\right]^{1 / 2}(\eta)\right\|^{2} & =\psi\left(\theta^{\varphi^{-1}}(\eta, \eta)\right)=\psi\left(R_{\eta}^{\varphi^{-1}} R_{\eta}^{\varphi^{-1}} *\right) \\
& =\psi(\varphi(\eta \otimes \eta))=(\psi \otimes \varphi)(\eta \otimes \eta) \\
& =(\psi \otimes \varphi)\left(R_{\eta}^{l} R_{\eta}^{l *}\right) \\
& =\left\|[\Delta(\psi \circ E \mid l)]^{1 / 2}(\eta)\right\|^{2} . \quad \text { Q.E.D. }
\end{aligned}
$$

2.6. Corollary. $E^{-1}=\sum_{i=1}^{\infty} 1 / \lambda_{i}$, where $\lambda_{i}$ are eigenvalue of $A$ with $\varphi(x)=$ $\operatorname{tr}(A x)$ for all $x \in N^{\prime}$.

Proof. It follows straightforwardly from Theorem 2.5 and Proposition 2.2. Q.E.D.

2.7. Corollary. Let $E$ be a nontrivial faithful normal conditional expectation of a type $I$ factor $M$ onto its subfactor $N$. Then $\operatorname{IND}_{E} \geq 4$.

Proof. Due to a theorem by Tomiyama [11], $N$ has to be of type $I$. It follows from Corollary 2.6 that $\mathrm{IND}_{E} \geq 4$. Q.E.D.

2.8. Let $E_{1}, E_{2}$ be two faithful normal conditional expectations of a type $I$ factor $M$ onto its subfactor $N$. Then $\operatorname{IND}_{E_{1}}=\mathrm{IND}_{E_{2}}$ if and only if $\sum 1 / \lambda_{i}=$ $\sum 1 / \gamma_{i}$, where $\left\{\lambda_{i}\right\}$ is the set of eigenvalues associated to $E_{1}$ and $\left\{\gamma_{i}\right\}$ is the set of eigenvalues associated to $E_{2}$ in Corollary 2.6. If there exists an automorphism $\theta$ of $M$, (which is actually inner, i.e., $\theta(x)=A d_{u}(x)$ for some unitary $u$ in $M$ ) such that $E_{1}=E_{2} \circ \theta$. Then $\left\{\lambda_{i}\right\}=\left\{\gamma_{i}\right\}$. Thus we can have uncountable family of nonconjugate faithful normal conditional expectations of type $I$ factor $M$ with the same index greater than 9 . In fact, one can easily construct such a family of $E$ 's on $M=M_{3}$ and $N=\mathscr{C}$, where $M_{3}$ is the factor of all $3 \times 3$ complex matrices, as follows.

Let

$$
A=\left[\begin{array}{lll}
\lambda_{1} & & 0 \\
& \lambda_{2} & \\
0 & & \lambda_{3}
\end{array}\right]
$$

with $\lambda_{i}>0, i=1,2,3$, and $\sum \lambda_{i}=1, \sum 1 / \lambda_{i}=\alpha>9$. A faithful normal state $\varphi_{A}$ on $M_{3}$ is defined by $\varphi_{A}(x)=\operatorname{tr}(A x)$. From Proposition 2.2 we have $\mathrm{IND}_{\varphi_{A}}=1 / \lambda_{1}+1 / \lambda_{2}+1 / \lambda_{3}=\alpha$. For each given $\alpha>9,\left\{\left(\lambda_{1}, \lambda_{2}\right) \mid 0<\lambda_{i}<1\right.$, $\left.i=1,2,0<\lambda_{1} \leq \lambda_{2} \leq 1-\lambda_{1}-\lambda_{2}<1 \& 1 / \lambda_{1}+1 / \lambda_{2}+1 / 1-\lambda_{1}-\lambda_{2}=\alpha\right\}$ is uncountable. Each such triple gives rise to a faithful normal state and these 
states are not pairwise unitarily equivalent. However, in the case of $2 \times 2$ matrix algebra, for any $\alpha \geq 4$ there can be at most two pairs of $\left(\lambda_{1}, \lambda_{2}\right)$ such that $0<\lambda_{i}<1, i=1,2, \lambda_{1}+\lambda_{2}=1$ and $1 / \lambda_{1}+1 / \lambda_{2}=\alpha$.

\section{INDEX OF TENSOR PRODUCTS}

3.1. Let $M$ be a factor of type $s$ and $N$ be a subfactor of type $t$. If there exists a normal conditional expectation of $M$ onto $N$ then $s \geq t$ by a theorem of Tomiyama [11].

Suppose that there is a faithful normal conditional expectation $E$ from $M$ onto $N$ with $\mathrm{IND}_{E}=4 \cos ^{2} \pi / n$ for $n>3$. Then the relative commutant of $N$ in $M$, denoted by $N^{C}$ is $\mathscr{C}[6,5]$. By a theorem due to Haagerup in [4], $E$ is the only faithful normal conditional expectation of $M$ onto $N$.

3.2. Let $E$ be a faithful normal conditional expectation of a factor $M$ onto its subfactor $N$, and let $R$ be a factor. We define a faithful normal conditional expectation $\widehat{E}$ of $M \otimes R$ onto $N \otimes R$ by $\widehat{E}(x \otimes r)=E(x) \otimes r$ for all $x \in M$, $r \in R$. Thus we show

3.3. Theorem. $\widehat{E}^{-1}(I)=E^{-1}(I)$.

The proof of the above theorem is based on the following propositions and lemmas.

Let $\varphi$ be a faithful normal semifinite weight on $B(\mathscr{H})$ (the same notation as in $\S 1)$. Then $\varphi(x)=\operatorname{tr}(A x)$ for all $x$ in $B(\mathscr{H})$, where $A$ is a positive definite densely-defined closed operator on $\mathscr{H}$. Let $\left\{\lambda_{i}\right\}$ be the set of all positive eigenvalues of $A$ and $\left\{\xi_{i}\right\}$ be corresponding orthonormal set of eigenvectors. Let $l$ be the identity functional on $\mathscr{C}$.

3.4. Lemma. $\Delta(\varphi \mid \imath)=A$ and $\Delta\left(\varphi^{-1} \mid \imath\right)=A^{-1}$.

Proof. For $\xi \in \mathscr{H},\left\|\Delta(\varphi \mid l)^{1 / 2} \xi\right\|^{2}=\varphi\left(\theta^{l}(\xi, \xi)\right)=\varphi(\xi \odot \xi)=\operatorname{tr}(A(\xi \odot \xi))=$ $\sum \lambda_{i}\left|\left\langle\xi, \xi_{i}\right\rangle\right|^{2}$. Thus $\Delta(\varphi \mid l)^{1 / 2}=A^{1 / 2}$ and $\Delta(\varphi \mid l)=A$. By [3, Theorem 9, condition $(3)]$ and $[6,1.2]$ we have

$$
\Delta\left(\varphi^{-1} \mid l\right)=\Delta\left(l \mid \varphi^{-1}\right)^{-1}=\Delta(\varphi \mid l)^{-1}=A^{-1} \text {. Q.E.D. }
$$

3.5. Proposition. Let $\varphi_{i}$ be faithful normal semifinite weights on $B\left(\mathscr{H}_{i}\right)$ for $i=1,2$. Then $\varphi_{1} \otimes \varphi_{2}$ is a faithful normal semifinite weight on $B\left(\mathscr{H}_{1}\right) \otimes B\left(\mathscr{H}_{2}\right) \simeq$ $B\left(\mathscr{H}_{1} \otimes H_{2}\right)$ and $\left(\varphi_{1} \otimes \varphi_{2}\right)^{-1}=\varphi_{1}^{-1} \otimes \varphi_{2}^{-1}$.

Proof. It is obvious that $\varphi_{1} \otimes \varphi_{2}$ is a faithful normal semifinite weight on $B\left(\mathscr{H}_{1}\right) \otimes B\left(\mathscr{H}_{2}\right)$, which is isomorphic to $B\left(\mathscr{H}_{1} \otimes \mathscr{H}_{2}\right)$. Let $\varphi_{i}(x)=\operatorname{tr}\left(A_{i} x\right)$ for $x \in B\left(\mathscr{H}_{i}\right)$, where $A_{i}$ is a positive definite densely-defined closed operator in $B\left(\mathscr{H}_{i}\right), i=1,2$. It is easy to see that $\left(\varphi_{1} \otimes \varphi_{2}\right)(x)=\operatorname{tr}\left(\left(A_{1} \otimes A_{2}\right) x\right)$ for $x$ in $B\left(\mathscr{H}_{1} \otimes H_{2}\right)$ and $\operatorname{tr}\left(x_{1} \otimes x_{2}\right)=\operatorname{tr}\left(x_{1}\right) \operatorname{tr}\left(x_{2}\right)$ for $x_{i}$ in $B\left(\mathscr{H}_{i}\right), i=1,2$. Then, it follows from Lemma 3.4 that $\Delta\left(\left(\varphi_{1} \otimes \varphi_{2}\right)^{-1} \mid \imath\right)=\left(A_{1} \otimes A_{2}\right)^{-1}$, which in turn is equal to $A_{1}^{-1} \otimes A_{2}^{-1}$ and $\Delta\left(\varphi_{i}^{-1} \mid \imath\right)=A_{i}^{-1}, i=1,2$. Since $\Delta(\varphi \mid \imath)$ determines $\varphi$ completely by Lemma $3.4,\left(\varphi_{1} \otimes \varphi_{2}\right)^{-1}=\varphi_{1}^{-1} \otimes \varphi_{2}^{-1}$. Q.E.D. 
Let $M_{i}$ be factors operating on $\mathscr{H}_{i}$, and $\varphi_{i}, \varphi_{i}^{\prime}$ be faithful normal semifinite weights on $M_{i}, M_{i}^{\prime}$ respectively, $i=1,2$. Then we show

3.6. Proposition. $\left(\varphi_{1} \otimes \varphi_{2}\right)^{-1}=\varphi_{1}^{-1} \otimes \varphi_{2}^{-1}$.

Proof. Let $l$ be the identity state on $\mathscr{C}$. By the commutation theorem of tensor product we know $M_{1}^{\prime} \otimes M_{2}^{\prime}=\left(M \otimes M_{2}\right)^{\prime}$ (see [9]). Since $\Delta\left(l\left(\varphi_{1} \otimes \varphi_{2}\right) \mid \varphi_{1}^{\prime} \otimes \varphi_{2}^{\prime}\right)=$ $\Delta\left(\imath \mid\left(\varphi_{1}^{\prime} \otimes \varphi_{2}^{\prime}\right)\left(\varphi_{1} \otimes \varphi_{2}\right)^{-1}\right)$, it suffices to show

$$
\Delta\left(l\left(\varphi_{1} \otimes \varphi_{2}\right) \mid \varphi_{1}^{\prime} \otimes \varphi_{2}^{\prime}\right)=\Delta\left(l \mid\left(\varphi_{1}^{\prime} \otimes \varphi_{2}^{\prime}\right)\left(\varphi_{1}^{-1} \otimes \varphi_{2}^{-1}\right)\right) .
$$

For arbitrary vector $\xi_{i} \in \mathscr{H}_{i}$, consider

$$
\begin{aligned}
& \left\|\Delta\left(l \mid \varphi_{1}^{\prime} \varphi_{1}^{-1} \otimes \varphi_{2}^{\prime} \varphi_{2}^{-1}\right)^{1 / 2}\left(\xi_{1} \otimes \xi_{2}\right)\right\|^{2} \\
& \quad=l\left(\theta^{\varphi_{1}^{\prime} \varphi_{1}^{-1} \otimes \varphi_{2}^{\prime} \varphi_{2}^{-1}}\left(\left(\xi_{1} \otimes \xi_{2}\right),\left(\xi_{1} \otimes \xi_{2}\right)\right)\right) \\
& \left.\quad=l\left(\varphi_{1}^{\prime} \varphi_{1}^{-1} \otimes \varphi_{2}^{\prime} \varphi_{2}^{-1}\right)^{-1}\left(\left(\xi_{1} \otimes \xi_{2}\right) \odot\left(\xi_{1} \otimes \xi_{2}\right)\right)\right) .
\end{aligned}
$$

It follows from Proposition 3.5 that

$$
\left(\varphi_{1}^{\prime} \varphi_{1}^{-1} \otimes \varphi_{2}^{\prime} \varphi_{2}^{-1}\right)^{-1}=\left(\varphi_{1}^{\prime} \varphi_{1}^{-1}\right)^{-1} \otimes\left(\varphi_{2}^{\prime} \varphi_{2}^{-1}\right)^{-1}=\varphi_{1} \varphi_{1}^{\prime-1} \otimes \varphi_{2} \varphi_{2}^{\prime-1}
$$

(the last equality is due to Haagerup in [4]). Thus, 3.6.1 becomes

$$
\begin{aligned}
l\left(\varphi_{1} \varphi_{1}^{\prime-1} \otimes \varphi_{2} \varphi_{2}^{\prime-1}\left(\left(\xi_{1} \otimes \xi_{2}\right) \odot\left(\xi_{1} \otimes \xi_{2}\right)\right)\right) \\
\quad=l\left(\varphi_{1} \otimes \varphi_{2}\right)\left(\varphi_{1}^{\prime-1} \otimes \varphi_{2}^{\prime-1}\right)\left(\left(\xi_{1} \otimes \xi_{2}\right) \odot\left(\xi_{1} \otimes \xi_{2}\right)\right) \\
\quad=l\left(\varphi_{1} \otimes \varphi_{2}\right)\left(\varphi_{1}^{\prime} \otimes \varphi_{2}^{\prime}\right)^{-1}\left(\left(\xi_{1} \otimes \xi_{2}\right) \odot\left(\xi_{1} \otimes \xi_{2}\right)\right), \quad \text { by Proposition 3.5 } \\
\quad=l\left(\varphi_{1} \otimes \varphi_{2}\right)\left(\theta^{\varphi_{1}^{\prime} \otimes \varphi_{2}^{\prime}}\left(\left(\xi_{1} \otimes \xi_{2}\right),\left(\xi_{1} \otimes \xi_{2}\right)\right)\right) \\
\quad=\left\|\Delta\left(l\left(\varphi_{1} \otimes \varphi_{2}\right) \mid \varphi_{1}^{\prime} \otimes \varphi_{2}^{\prime}\right)^{1 / 2}\left(\xi_{1} \otimes \xi_{2}\right)\right\|^{2} .
\end{aligned}
$$

Hence $\Delta\left(l\left(\varphi_{1} \otimes \varphi_{2} \mid \varphi_{1}^{\prime} \otimes \varphi_{2}^{\prime}\right)=\Delta\left(l \mid\left(\varphi_{1}^{\prime} \otimes \varphi_{2}^{\prime}\right)\left(\varphi_{1}^{-1} \otimes \varphi_{2}^{-1}\right)\right)\right.$. Q.E.D.

3.7. Lemma. $(E \otimes \mathrm{id})^{-1}=E^{-1} \otimes \mathrm{id}$.

Proof. Coming back to the setup in 3.2, we let $\varphi, \varphi_{r}$ be faithful normal states on $M^{\prime}, R^{\prime}$ respectively, and $\psi, \psi_{r}$ be faithful normal states on $N, R$ respectively. Since $\Delta\left(\left(\psi \otimes \psi_{r}\right)(E \otimes\right.$ id $\left.) \mid \varphi \otimes \varphi_{r}\right)=\Delta\left(\psi \otimes \psi_{r} \mid\left(\varphi \otimes \varphi_{r}\right)(E \otimes \text { id })^{-1}\right)$ and the equation determines $(E \otimes \text { id })^{-1}$ completely from $E \otimes$ id, it suffices to show $\Delta\left(\left(\psi \otimes \psi_{r}\right)(E \otimes \mathrm{id}) \mid \varphi \otimes \varphi_{r}\right)=\Delta\left(\psi \otimes \psi_{r} \mid\left(\varphi \otimes \varphi_{r}\right)\left(E^{-1} \otimes \mathrm{id}\right)\right)$. For any vectors $\eta_{i} \in \mathscr{H}_{i}, i=1,2$, where $M, N \subseteq B\left(\mathscr{H}_{1}\right)$ and $R \subseteq B\left(\mathscr{H}_{2}\right)$, consider

$$
\begin{aligned}
& \left\|\Delta\left(\left(\psi \otimes \psi_{r}\right)(E \otimes \mathrm{id}) \mid \varphi \otimes \varphi_{r}\right)^{1 / 2}\left(\eta_{1} \otimes \eta_{2}\right)\right\|^{2} \\
& \quad=\left(\psi \circ E \otimes \psi_{r}\right)\left(\theta^{\varphi \otimes \varphi_{r}}\left(\eta_{1} \otimes \eta_{2}, \eta_{1} \otimes \eta_{2}\right)\right) \\
& \quad=\left(\psi \circ E \otimes \psi_{r}\right)\left(\left(\varphi \otimes \psi_{r}\right)^{-1}\left(\left(\eta_{1} \otimes \eta_{2}\right) \odot\left(\eta_{1} \otimes \eta_{2}\right)\right)\right) .
\end{aligned}
$$


It follows from Proposition 3.6 that $\left(\varphi \otimes \varphi_{r}\right)^{-1}=\varphi^{-1} \otimes \varphi_{r}^{-1}$ and 3.7.1 becomes

$$
\begin{aligned}
(\psi \circ E & \left.\otimes \psi_{r}\right)\left(\left(\varphi^{-1} \otimes \varphi_{r}^{-1}\right)\left(\left(\eta_{1} \otimes \eta_{2}\right) \odot\left(\eta_{1} \otimes \eta_{2}\right)\right)\right) \\
& =\left(\psi \otimes \psi_{r}\right)\left(E \circ \varphi^{-1} \otimes \varphi_{r}^{-1}\right)\left(\left(\eta_{1} \otimes \eta_{2}\right) \odot\left(\eta_{1} \otimes \eta_{2}\right)\right) \\
& =\left(\psi \otimes \psi_{r}\right)\left(\left(\varphi \circ E^{-1}\right)^{-1} \otimes \varphi_{r}^{-1}\right)\left(\left(\eta_{1} \otimes \eta_{2}\right) \odot\left(\eta_{1} \otimes \eta_{2}\right)\right) \\
& =\left(\psi \otimes \psi_{r}\right)\left(\varphi \circ E^{-1} \otimes \varphi_{r}\right)^{-1}\left(\left(\eta_{1} \otimes \eta_{2}\right) \odot\left(\eta_{1} \otimes \eta_{2}\right)\right), \quad \text { by Proposition 3.6, } \\
& =\left(\psi \otimes \psi_{r}\right)\left(\theta^{\varphi \circ E^{-1} \otimes \varphi_{r}}\left(\eta_{1} \otimes \eta_{2}, \eta_{1} \otimes \eta_{2}\right)\right) \\
& =\left\|\Delta\left(\psi \otimes \psi_{r} \mid\left(\varphi \otimes \varphi_{r}\right)\left(E^{-1} \otimes \mathrm{id}\right)\right)^{1 / 2}\left(\eta_{1} \otimes \eta_{2}\right)\right\|^{2} .
\end{aligned}
$$

Hence $\Delta\left(\left(\psi \otimes \psi_{r}\right)(E \otimes\right.$ id $\left.) \mid \varphi \otimes \varphi_{r}\right)=\Delta\left(\psi \otimes \psi_{r} \mid\left(\varphi \otimes \varphi_{r}\right)\left(E^{-1} \otimes\right.\right.$ id $\left.)\right)$. Q.E.D.

3.8. Now, it is obvious that Theorem 3.3 follows easily from Lemma 3.7.

3.9. Let $M$ be the hyperfinite $\mathrm{II}_{1}$ factor and $N$ its subfactor with the index $[M, N] \in\left\{4 \cos ^{2} \pi / n \mid n \geq 3\right\} \cup[4, \infty)$, where $E$ is the trace preserving faithful normal conditional expectation of $M$ onto $N$. Let $R$ be a type III $_{\lambda}$ factor, then we exhibit a pair of type $\operatorname{III}_{\lambda}$ factors $M \otimes R$ and $N \otimes R, 0 \leq \lambda \leq 1$, and a faithful normal conditional expectation $\widehat{E}=E \otimes$ id such that $\operatorname{IND}_{\widehat{E}}=$ $\mathrm{IND}_{E}=\left\{4 \cos ^{2} \pi / n \mid n \geq 3\right\} \cup[4, \infty)$. In the case $[M, N]=\mathrm{IND}_{E}=4 \cos ^{2} \pi / n$ for $n \geq 3$, then $E$ is the only faithful normal conditional expectation of $M$ onto $N$.

3.10. Theorem. Let $E_{i}$ be a faithful normal conditional expectation from a factor $M_{i}$ onto its subfactor $N_{i}, i=1,2$. Then $\mathrm{IND}_{E_{1} \otimes E_{2}}=\left(\mathrm{IND}_{E_{1}}\right)\left(\mathrm{IND}_{E_{2}}\right)$, where $E_{1} \otimes E_{2}$ is a faithful normal conditional expectation of $M_{1} \otimes M_{2}$ into $N_{1} \otimes N_{2}$.

Proof. Denote $E_{1} \otimes$ id, (id $\otimes E_{2}$ respectively) by $\widehat{E}_{1},\left(\widehat{E}_{2}\right.$ respectively), and then $E_{1} \otimes E_{2}=\widehat{E}_{1} \circ \widehat{E}_{2}$ and $\left(E_{1} \otimes E_{2}\right)^{-1}=\widehat{E}_{2}^{-1} \circ \widehat{E}_{1}^{-1}$. By Theorem 3.3, we have $\left(E_{1} \otimes E_{2}\right)^{-1}(I)=\widehat{E}_{2}^{-1}\left(\widehat{E}_{1}^{-1}(I)\right)=\widehat{E}_{2}^{-1}\left(E_{1}^{-1}(I) I\right)=E_{1}^{-1}(I) \widehat{E}_{2}^{-1}(I)=$ $E_{1}^{-1}(I) E_{2}^{-1}(I)$. In fact, $\widehat{E}_{2}^{-1}(I)=E_{2}^{-1}(I)$ follows from a proof symmetric to that of Theorem 3.3. Q.E.D.

3.11. Let $E_{\alpha}$ be an uncountable family of nonconjugate faithful normal conditional expectations of a type I factor $M$ onto its subfactor $N$ considered in 2.8. For any factor $R$ of type $s, s=\mathrm{I}$ or II or III, $M \otimes R$ is of type $s$ and $\left\{E_{\alpha} \otimes \mathrm{id}\right\}$ is an uncountable family of nonouterconjugate faithful normal conditional expectations of $M \otimes R$ onto $N \otimes R$ with the same index. Similar works on an uncountable family of conjugacy classes of shifts with the same index on a type $\mathrm{II}_{1}$-factor have recently been discussed in $[2,8]$.

3.12. Concluding remarks. For any $4 \leq \lambda<9$ it is still unknown to the author how to find a pair of $\mathrm{II}_{1}$-factors $M \supset N$ such that there are uncountable nonisomorphic faithful normal conditional expectations $E_{\alpha}$ 's with $\operatorname{IND}_{E_{r}}=\lambda$. 


\section{REFERENCES}

1. D. Bures and H.-S. Yin, Shifts on the hyperfinite factor of type $\mathrm{II}_{1}$, preprint.

2. M. Choda, Shifts of the hyperfinite $\mathrm{II}_{1}$-factors, J. Operator Theory 17 (1987), 223-235.

3. A. Connes, Spatial theory of von Neumann algebras, J. Funct. Anal. 35 (1980), 153-164.

4. U. Haagerup, Operator valued weights in von Neumann algebras I, II, J. Funct. Anal. 32 (1979). (175-206; 33 (1979), 339-361.)

5. V. Jones, Index for subfactors, Invent. Math. 72 (1983), 1-25.

6. H. Kosaki, Extension of Jones' index theory to arbitrary factors, J. Funct. Anal. 66 (1986), 123-140.

7. F. J. Murray and J. von Neumann, On rings of operators, Ann. of Math. 37 (1936), 116-229.

8. R. T. Powers, An index theory for semigroups of *-endomorphisms of $B(\mathscr{H})$ and type $\mathrm{II}_{1}$-factors, Contemp. Math. vol. 62, Amer. Math. Soc., Providence, RI, 1987, pp. 447-460.

9. M. Rieffel and A. van Daele, The commutation theorem for tensor products of von Neumann algebras, Bull. London Math. Soc. 7 (1975), 257-260.

10. S. Strătilă, Modular theory in operator algebras, Abacus Press, 1981.

11. J. Tomiyama, Tensor product and projections of norm one in von Neumann algebras, Lecture Notes, Kobenhavn Univ., 1971.

Department of Mathematical Science, Oakland University, Rochester, Michigan 48309 